\title{
Pressure-enthalpy coupling for subsonic flows with density variation
}

\author{
M. Emans ${ }^{1}, \mathrm{~S}$. Frolov $^{2}$, B. Lidskii ${ }^{2}$, V. Posvyanskii ${ }^{2}$, \\ Z. Žunič ${ }^{3}$ \& B. Basara ${ }^{3}$ \\ ${ }^{1}$ IMCC, Linz, Austria \\ ${ }^{2}$ N. N. Semenov Institute of Chemical Physics, Moscow, Russia \\ ${ }^{3}$ AVL List GmbH, Graz, Austria
}

\begin{abstract}
The method we present is derived from the SIMPLE algorithm. Instead of solving the linear equations for each variable and the pressure-correction equation separately in a so called segregated manner it relies on the solution of a linear system that comprises the discretisation of enthalpy and pressure-correction equation that are linked through physical coupling terms. These coupling terms are deduced from a more accurate approximation of the density update (compared to standard SIMPLE method) that is reasonable with respect to the thermodynamic formulation of the density increment. The method leads to a considerable acceleration of the non-linear SIMPLE convergence not only in cases characterised by a global density change due to the rise of the pressure, but also if the enthalpy is merely spatially distributed in the computational domain. In this contribution we will first discuss the key idea of the new method and compare it to established techniques. Then we will demonstrate its advantages in terms of computational efficiency and robustness for industrial CFD applications, e.g. combustion and engine flow calculations on unstructured grids.
\end{abstract}

Keywords: finite volumes, pressure-enthalpy coupling, variable-density flow, CFD.

\section{Introduction}

Robust methods that provide an appropriate approximation of the solution of the Navier-Stokes equations for CFD (computational fluid dynamics) applications employ finite volumes for the spatial discretisation and master the non-linearity of 
the system as well as the coupling of the momentum equations and the continuity equation by iterative algorithms derived from SIMPLE ("semi-implicit method for pressure-linked equations"); they are used to calculate the velocity and the pressure field.

Many ways have been proposed to accelerate the convergence of the SIMPLE algorithm in the genuine formulation of Patankar and Spalding [1] that tends to be unsatisfactory in some situations. Among the most important ones are SIMPLER, SIMPLEC, and PISO, see van Doormal and Raithby [2] for an overview. Whereas genuine SIMPLE requires the solution of one linear system per unknown and time step, the enhanced methods require the solution of more than one linear system per unknown and time step. The size of the systems always corresponds to the number of finite volumes; despite occasional convergence problems these methods are applied with great success in many important engineering applications.

Coupling equations and calculating two or more variables at the same time is an alternative to obtain accelerated convergence compared to the established approaches that are referred to as segregated. It seems, however, that until very recently one tends to prefer segregated approaches in CFD simulation tools for engineering applications On the other hand, long references for methods that couple momentum equations and continuity equation can be easily listed, e.g. Tai et al. [3] and Chen et al. [4]. The increased availability of parallel computers with large memory makes it possible and attractive to apply methods with coupled solution of linear systems to practical engineering problems. This includes methods that couple other equations than momentum equations and continuity equation.

For compressible flows the pressure-correction equation (that represents the mass conservation in SIMPLE algorithm) depends not only on the velocity field, but also on the density field, see Demirdžić et al. [5]. The segregated methods improve velocity update with respect to the mass conservation, but the error introduced by the density field, calculated based on the pressure of the previous iteration, is not reduced. Since the density depends strongly on the temperature, this error can be large if the actual guess of the temperature is not yet close to the solution. As a matter of fact the right-hand-side of the pressure-correction equation strongly depends on the enthalpy changes through the density field and, on the other hand, the pressure change as a result of this pressure-correction equation contributes significantly to the right-hand side of the enthalpy equation. A stronger link between enthalpy and pressure allows to employ a better approximation to the density in the pressure-correction equation. This motivated us to couple these two equations within the context of the SIMPLE algorithm.

\section{Conventional SIMPLE method}

In this contribution we consider a finite volume approach with collocated variable arrangement for the spacial discretisation of the governing equations and an implicit first order time discretisation. The discretised momentum, continuity, and energy equations for the discrete unknown velocity field $\vec{u}$, the pressure field $\vec{p}$, 
and the (static) enthalpy field $\vec{h}$ read

$$
\begin{aligned}
A(\hat{\vec{u}}) \vec{u}+M \vec{p} & =\vec{b} \\
C \hat{\vec{u}} & =\vec{c} \\
G(\hat{\vec{u}}) \vec{h} & =\tilde{\vec{g}} .
\end{aligned}
$$

Here, $A$ denotes the discretised and linearised operator that acts on the velocity field in the momentum equations, i.e. it expresses convective, diffusive, and inertia components. $M$ is the discretisation of the pressure term, $\vec{b}$ the body force term, $C$ represents the discretised continuity equation, $\vec{c}$ the corresponding right-hand side; the energy equation is represented by $G$ and $\tilde{\vec{g}}$. The hat $\left(^{\wedge}\right)$ on top of the vectors indicates that the vector is discretised on the grid that corresponds to the cell faces. The discretisation of the operators requires that both, the velocity in the cell centre $\vec{u}$ and the velocity at the cell faces $\hat{\vec{u}}$ appear in the system of equations. Both variables are linked by a linear interpolation operator $S$. The above system is completed by a relation between density and two thermodynamic variables, e.g. pressure and temperature

$$
\varrho=\frac{p}{R \cdot T}
$$

where $R$ is the gas constant and $T=T(h)$.

The iterative SIMPLE algorithm uses updates for pressure and velocity, $\vec{p}^{\prime}=$ $\vec{p}^{(m)}-\vec{p}^{(m-1)}$ and $\vec{u}^{\prime}=\vec{u}^{(m)}-\vec{u}^{*}$ where $m$ denotes the iteration count and $\vec{u}^{*}$ is the solution of the momentum equations with the pressure taken from the previous iteration. A stable and robust algorithm for collocated grids is obtained if the technique of Rhie and Chow [6] is applied to the formulation of the pressurecorrection equation. For this we introduce the operator

$$
\hat{A}_{M}:=\left[S A_{D}^{-1} \overrightarrow{1}\right]
$$

where $A_{D}:=\operatorname{diag}(A)$ and the square brackets $[\vec{x}]$ denote a diagonal matrix that has the element of value $x_{j}$ ( $j$-th component of vector $\vec{x}$ ) in the $j$-th row, i.e. $[\vec{x}]=\operatorname{diag}\left(\overrightarrow{1} \vec{x}^{T}\right)$. The pressure-correction equation can be derived, see Demirdžić et al. [5], as

$$
\begin{aligned}
\left\{C _ { c } \left(\left[\hat{\vec{\varrho}}^{(m-1)}\right]\left(-\hat{A}_{M} \hat{M}\right)\right.\right. & \left.\left.+\left[\hat{\vec{u}}^{*}\right] S[\vec{r}]\right)+[\vec{o}][\vec{r}]\right\} \vec{p}^{\prime}= \\
& -[\vec{o}]\left(\vec{\varrho}^{(m-1)}-\vec{\varrho}_{0}\right)-C_{c}\left[\hat{\vec{\varrho}}^{(m-1)}\right] \hat{\vec{u}}^{*}
\end{aligned}
$$

where the vector $\vec{o}$ is composed of $o_{j}=V_{j} / \delta t$ with the cell volume $V_{j}$ and the time step $\delta t$, the vector $\vec{r}$ contains the components $r_{j}=1 /\left(R_{j} \cdot T_{j}\right)$ and the continuity operator is split as $C \equiv C_{c}[\hat{\vec{\varrho}}] . \varrho_{0}$ is the density of the previous time step. The pressure-correction equation contains as a central assumption the relation

$$
\hat{\vec{u}}^{\prime}=-\hat{A}_{M} \hat{M} \vec{p}^{\prime}
$$

that uses the gradient operator $\hat{M}$ on the cell faces. 
The segregated SIMPLE algorithm for compressible flows with temperature variation on collocated grids is:

1. compute density field $\vec{\varrho}^{(m-1)}$ using $\vec{p}^{(m-1)}$ and $\vec{T}^{(m-1)}$ with eqn. (4) or, alternatively, from tabulated material data

2. evaluate $A$ in eqn. (1) with $\hat{\vec{u}}^{(m-1)}$ and solve for $\vec{u}=\vec{u}^{*}$

3. interpolate $\vec{\varrho}^{(m-1)}$, accumulate left-hand side operator of eqn. (6), compute right-hand side and solve for $\vec{p}^{\prime}$

4. compute $\hat{\vec{u}}^{\prime}$ with eqn. (7)

5. update $\hat{\vec{u}}$ and $\vec{p}$ to obtain $\hat{\vec{u}}^{(m)}$ and $\vec{p}^{(m)}$

6. compute coefficients and solve eqn. (3) for $h^{(m)}$

\section{Pressure-enthalpy coupling}

Apart from the special actions taken for pressure and velocity that have been highlighted above, in a segregated approach, the solution of a coupled system is approached iteratively. For each unknown, a linear system

$$
B \vec{\phi}=\vec{b}
$$

is solved; the coupling is achieved through the usage of the latest available values for the computation of the matrix coefficients and the right-hand sides. This is referred to as segregated approach. Coupling $n$ variables, on the other hand, means that systems like

$$
\left(\begin{array}{cccc}
B_{11} & B_{12} & \cdots & B_{1 n} \\
B_{21} & B_{22} & \cdots & B_{2 n} \\
\vdots & \cdots & \ddots & \vdots \\
B_{n 1} & \cdots & \cdots & B_{n n}
\end{array}\right)\left(\begin{array}{c}
\vec{\phi}_{1} \\
\vec{\phi}_{2} \\
\vdots \\
\vec{\phi}_{n}
\end{array}\right)=\left(\begin{array}{c}
\vec{b}_{1} \\
\vec{b}_{2} \\
\vdots \\
\vec{b}_{n}
\end{array}\right)
$$

are solved. The matrices on the main diagonal are identical to those matrices that are used in the segregated approach. The off-diagonal matrices reflect the coupling, i.e. the mutual influences.

From thermodynamics the relation

$$
\vec{\varrho}^{\prime}=[\vec{r}]\left(\vec{p}^{\prime}+[\vec{k}] \vec{h}^{\prime}\right)
$$

is derived where the components of $\vec{k}$ are $k=-p /\left(c_{p} \cdot T\right)$. Note that the difference to standard SIMPLE is that in the latter the second term (or an equivalent contribution) is neglected. If now the enthalpy equation is equivalently reformulated for an enthalpy update $\vec{h}^{\prime}=\vec{h}^{(m)}-\vec{h}^{(m-1)}$ and coupled to the pressure-correction equation such that in eqn. (9) $n=2$, the four needed matrices can be determined in the following way:

$$
\begin{aligned}
& B_{11}=C_{c}\left(\left[\hat{\vec{\varrho}}^{(m-1)}\right]\left(-\hat{A}_{M} \hat{M}\right)+\left[\hat{\vec{u}}^{*}\right] S[\vec{r}]\right)+[\vec{o}][\vec{r}] \\
& B_{12}=C_{c}\left[\hat{\vec{u}}^{*}\right] S[\vec{r}][\vec{k}]+[\vec{o}][\vec{r}][\vec{k}]
\end{aligned}
$$




$$
\begin{aligned}
& B_{21}=-[\vec{o}] \\
& B_{22}=G
\end{aligned}
$$

The right-hand side vectors are

$$
\begin{aligned}
& \vec{b}_{1}=-[\vec{o}]\left(\vec{\varrho}^{(m-1)}-\vec{\varrho}_{0}\right)-C_{c}\left[\hat{\vec{\varrho}}^{(m-1)}\right] \hat{\vec{u}}^{*} \\
& \vec{b}_{2}=\vec{g}+[\vec{o}]\left(\vec{p}^{(m-1)}-\vec{p}_{0}\right)
\end{aligned}
$$

where $\vec{g}=\tilde{\vec{g}}-[\vec{o}]\left(\vec{p}^{(m)}-\vec{p}_{0}\right)$ and $\vec{p}_{0}$ is the pressure of the previous time step.

This is integrated into the SIMPLE algorithm for compressible flows. For the pressure-enthalpy coupling scheme, step 3 of above form of this algorithm has been replaced by

3. interpolate $\vec{\varrho}^{(m-1)}$, accumulate left-hand side operator for $n=2$ of eqn. (9) using the relations (11), (12), (13), and (14), compute right-hand side according to relations (15) and (16) and solve for $\phi_{1}=\vec{p}^{\prime}$ and $\phi_{2}=\vec{h}^{\prime}$ and step 5 by

5. update $\hat{\vec{u}}, \vec{p}$, and $\vec{h}$ to obtain $\hat{\vec{u}}^{(m)}, \vec{p}^{(m)}$ and $\vec{h}^{(m)}$. Step 6 has become obsolete.

\section{Numerical experiments}

The pressure-enthalpy coupling has been implemented into the CFD package FIRE $^{(R)}$ (distributed by AVL) that uses the SIMPLE method as the standard algorithm. It has been demonstrated in Emans et al. [7] that the pressure-enthalpy coupling accelerates the SIMPLE method by a factor of up to five. While the examples in that publication were rather simple in terms of geometry, we will demonstrate here the efficiency of the pressure-enthalpy scheme in a simulation of industrial relevance.

The implementation of the SIMPLE method is that of a commercial code. The employed discretisation practice was explored in other publications starting with Demirdžić and Muzaferija [8], and continuing with Ferziger and Perić [9], Marthur and Murthy [10], Basara [11], Basara et al. [12] etc. Hence the method has been applied and proved on various applications and a comparison of the obtained results to measurements or to analytical solutions is skipped here. We observe throughout that the proposed pressure-enthalpy coupling method yields essentially the same results as the SIMPLE method.

The pressure-correction equation of the segregated SIMPLE is solved by an AMG-preconditioned conjugate gradient method where the AMG is of Smoothed Aggregation type of Vaněk et al. [13], for details about the implementation see Emans [14]. The coupled system is solved by an AMG-preconditioned GMRES that is restarted each 10 iterations. The preconditioning is done as v-cycle with two Gauß-Seidel sweeps for both, pre- and post-smoothing. The grid hierarchy is setup by aggregation of pairs where the selection of the pairs follows the algorithm described in detail by Notay [15]. All other non-symmetric systems are 
solved by a BiCGstab algorithm that is preconditioned by an incomplete Cholesky factorisation.

\subsection{Test cases from the simulation of an engine cycle}

Our two benchmark cases are short but representative periods taken from an unsteady simulation of a full cycle of a four cylinder gasoline engine. The variation of the position of the piston during the engine cycle as well as some characteristic results of the simulation are shown in figure 1.

The simulation comprises the gas flow and the combustion in one of the cylinders. The stroke of the cylinder is $81.4 \mathrm{~mm}$, the bore is $79.0 \mathrm{~mm}$ yielding a (maximum) volume of $0.4 l$ (per cylinder). Each benchmark case consists of a few time-steps. The three-dimensional computational domain is subject to change in time: It contains the interior of the cylinder and the parts of the ducts through which the air is sucked into the cylinder or hot gas is expelled from it. The piston surface is a moving boundary. The engine runs at $5500 \mathrm{rpm}$. A three-dimensional simulation of a full engine cycle comprises the simulation of the (compressible) flow of cold air into the cylinder while the piston is moving downward, the subsequent compression after the valves are closed, the combustion of the explosive mixture, and the discharge of the hot gas while the piston moves upward. In the simulation the modelling by the above discussed system of equations is amended by a standard $\mathrm{k}-\varepsilon$ turbulence model; the fluid properties are those of air. The model fuel that is burnt is octane; an eddy break-up model is used to simulate the combustion process. The simulation is carried out with the FIRE $^{(R)}$ code that has been validated for this type of problem many times, see e.g. Priesching et al. [16]. Further information about the setup can be found in Emans [17].

Example 1a comprises 4 degree crank angle $\alpha$ within the compression phase, i.e. we observe a closed system with adiabatic walls; the unstructured mesh consists of 238000 mostly hexagonal cells. This period is simulated with four different

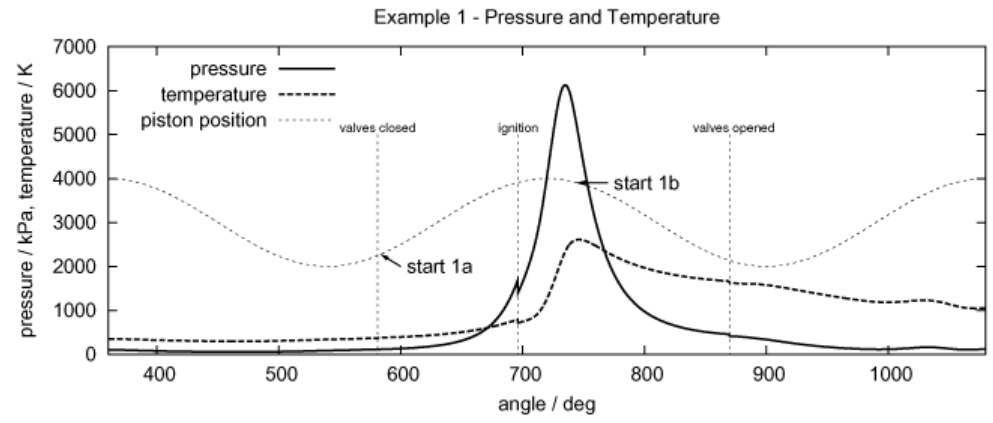

Figure 1: Scheme of the engine cycle and characteristic simulation results; the arrows point to the start of the benchmark cases. 

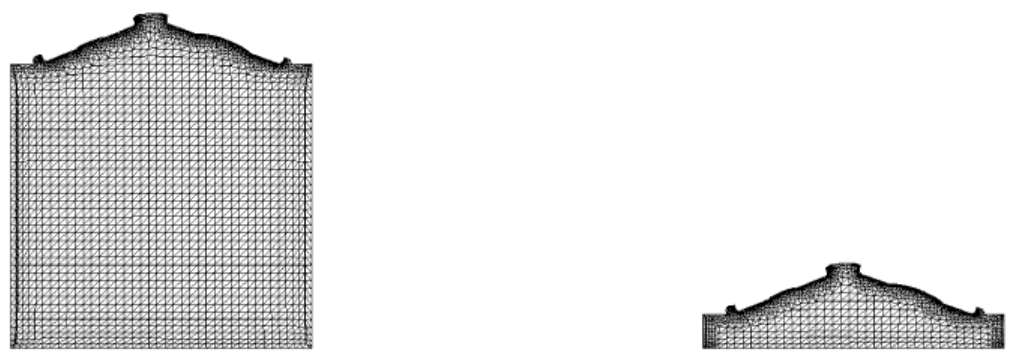

Figure 2: Slices through the first meshes of the benchmark cases: example 1a (compression) right, example $1 \mathrm{~b}$ (combustion) left.

time resolutions: $\delta \alpha_{1}=0.4^{\circ}, \delta \alpha_{2}=0.2^{\circ}, \delta \alpha_{3}=0.05^{\circ}$, and $\delta \alpha_{4}=0.01^{\circ}$ This corresponds to time steps of $\delta t_{1}=1.2 \cdot 10^{-6} s, \delta t_{2}=0.60 \cdot 10^{-6} s$, $\delta t_{3}=0.15 \cdot 10^{-6} s \delta t_{4}=0.03 \cdot 10^{-6} s$; with these time steps we cover the range that is relevant for applications in engineering. Each calculation has been carried out once with segregated SIMPLE (SIM) that uses block-pressure adjustment, see Ahmadi-Befrui [18], and with SIMPLE with pressure-enthalpy coupling (PH). The hardware consisted of one node of a Linux-cluster equipped with 2 quad-cores (Intel Xeon CPU X5365, 3.00GHz, main memory $16 \mathrm{~GB}$, L1-cache $2.4 .32 \mathrm{kB}$, L2-cache 2.4 MB).

Example 1b comprises 4 degree crank angle within the combustion phase, i.e. here, additionally to fluid flow, combustion is modelled; the unstructured mesh consists of 254000 mostly hexagonal cells. Other settings as well as the hardware are identical. The first meshes used in example $1 \mathrm{a}$ and example $1 \mathrm{~b}$ are shown in figure 2 .

\subsection{Convergence and performance}

The data shown in figure 3 demonstrates that the pressure-enthalpy coupling improves the convergence of SIMPLE significantly in all cases. The computing time is reduced to a lower extent than the number of iterations since the solution of the coupled system is more expensive than the solution of the systems in the segregated approach. For the largest part this is due to the more expensive solution of the linear system: The pressure-correction equation is a semi-definite or definite system in the case of the conventional SIMPLE algorithm, but it has no beneficial properties apart from the sparseness that can be exploited by the solver in the case of pressure-enthalpy coupling algorithm. In most cases, however, the computing time is still reduced significantly. The exception is the coarsest time resolution of example $1 b$.

It can be seen from the data in figure 4 that the savings in terms of SIMPLE iterations increase with decreased time step. The savings range from $63 \%(\delta \alpha=$ $0.01^{\circ}$, example $\left.1 \mathrm{~b}\right)$ to $26 \%\left(\delta \alpha=0.4^{\circ}\right.$, example $\left.1 \mathrm{~b}\right)$. The maximum saving in 

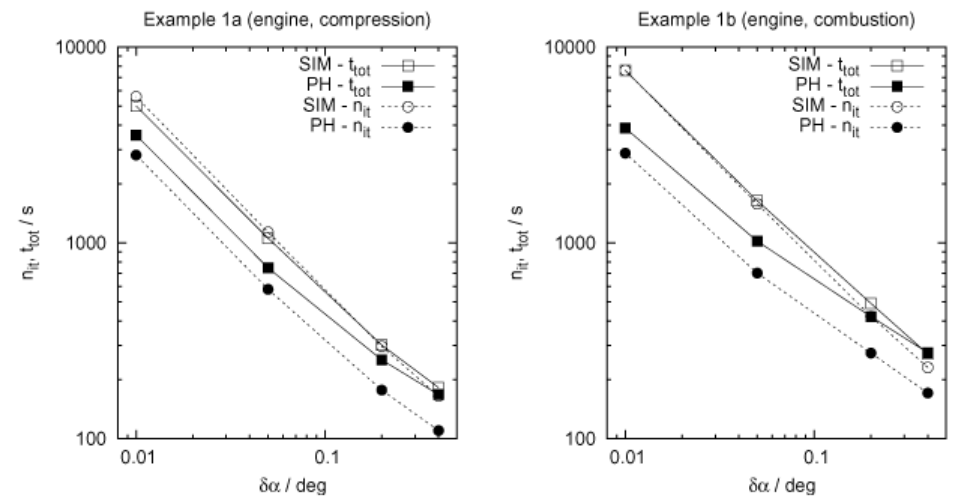

Figure 3: Total computing time $t_{t o t}$ and number of SIMPLE-iterations $n_{i t}$ of the benchmarks of examples $1 \mathrm{a}$ and $1 \mathrm{~b}$.
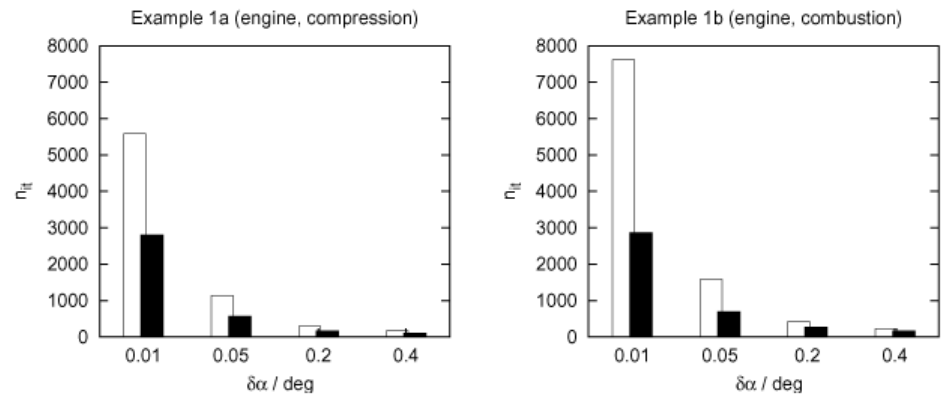

Figure 4: Comparison or the number of SIMPLE iterations $n_{i t}$ of the benchmarks of examples $1 \mathrm{a}$ and $1 \mathrm{~b}$.

terms of computing time is $49 \%$ ( $\delta \alpha=0.01^{\circ}$, example $\left.1 \mathrm{~b}\right)$. Note that in Emans et al. [7] the maximum acceleration through the pressure-enthalpy coupling is obtained in cases where the segregated SIMPLE algorithm could not be amended by block-pressure adjustment. The acceleration observed here confirms the data reported in Emans et al. [7] for the comparison between SIMPLE without blockpressure adjustment and pressure-enthalpy coupling.

\section{Conclusions}

The presented pressure-enthalpy coupling scheme accelerates the convergence of the SIMPLE algorithm significantly. The method is particularly efficient if small time steps are chosen. The disadvantage of the method is that the computational effort to solve linear systems for the pressure correction mechanism is increased 
compared to segregated SIMPLE. Despite this we can show that the computations of a complex engine simulation using the pressure-enthalpy coupling scheme are faster by a factor of up to two compared to those of segregated SIMPLE.

\section{References}

[1] Patankar S.V. \& Spalding, D.B., A calculation procedure for heat, mass and momentum transfer in three-dimensional parabolic flows, International Journal Heat Mass Transfer, 15, pp. 1787-1806, 1972

[2] van Doormal J.P. \& Raithby G.D., Enhancements of the SIMPLE Method for Predicting Incompressible Flows, Numerical Heat Transfer, 7, pp. 147-163, 1984

[3] Tai, C.H., Zhou, Y. \& Liew, K.M., Parallel-multigrid computations of unsteady incompressible viscous flows using a matrix-free implicit method and high-resolution characteristics-based scheme, Computational Methods in Applied Mechanics and Engineering, 194, pp. 3949-3983, 2005

[4] Chen, Z.J., Marella S.V. \& Przekwas A.J., A finite volume method of pressure-coupled solver for incompressible/compressible flows, Proc. of the $47^{\text {th }}$ AIAA Aerospace Sciences Meeting Including The New Horizons Forum and Aerospace Exposition, AIAA 2009-600, Orlando, Florida (USA), 2009

[5] Demirdžić I., Lilek Ž. \& M. Perić, A Collocated Finite Volume Method for Predicting Flows at All Speeds, International Journal for Numerical Methods in Fluids, 16, pp. 1029-1050, 1993

[6] Rhie, C.M. \& Chow, W.L., Numerical Study of the Turbulent Flow past an Airfoil with Trailing Edge Separation, AIAA Journal, 21, pp. 1525-1532, 1983

[7] Emans M., Frolov, S., Lidskii, B. Posvyanskii, V. \& Basara, B., A finite volume pressure-enthalpy coupling scheme for compressible flows, Journal of Computational Physics, submitted

[8] Demirdžić, I. \& Muzaferija, S., Numerical Method for Coupled Fluid Flow, Heat Transfer and Stress Analysis Using Unstructured Moving Meshes With Cells of Arbitrary Topology, Computational Methods in Applied Mechanical Engineering 125, pp. 235-255, 1995

[9] Ferziger, J.H. \& and Perić, M., Computational Methods for Fluid Dynamics, Springer-Verlag: Berlin and New York, 1996

[10] Marthur, S.R. \& Murthy, J.Y., A Pressure Based Method for Unstructured Meshes, Numerical Heat Transfer B 31, pp. 195-215, 1997

[11] Basara, B., Employment of the second-moment turbulence closure on arbitrary unstructured grids, International Journal for Numerical Methods in Fluids, 44, pp. 377-407, 2004

[12] Basara, B., Alajbegovic, A., \& Beader, D., Simulation of single- and twophase flows on sliding unstructured meshes using finite volume method, International Journal for Numerical Methods in Fluids, 45, pp. 1137-1159, 2004 
[13] Vaněk, P., Brezina M. \& Mandel, J., Algebraic Multigrid by Smoothed Aggregation for Second and Fourth Order Elliptic Problems, Computing, 56, pp. 179-196, 1996

[14] Emans M.: Performance of Parallel AMG-Preconditioners in CFD-Codes for Weakly Compressible Flows, Parallel Computing, 36, pp. 326-338, 2010

[15] Notay, Y., An aggregation-based algebraic multigrid method, Electronic Transactions on Numerical Analysis, 37, pp. 123-146, 2010

[16] Priesching P., Wanker R., Cartellieri P. \& Tatschl R., CFD Modelling of HCCI Engine Combustion - Validation and Application, Proceedings of ICE 2003, Capri, 2003

[17] Emans M.: AMG for linear systems in engine flow simulations, Proceedings of PPAM09, Lecture Notes in Computer Science, Springer Verlag: Berlin and New York, 2010, to appear

[18] Ahmadi-Befrui, B., Analysis of flow evolution in the cylinders of motored reciprocating engines, $\mathrm{PhD}$ Thesis, Imperial College of Science \& Technology, University of London, 1985 